(C) 2002 International Press

Adv. Theor. Math. Phys. 6 (2002) 575-591

\title{
Topological Quantum Field Theory
}

\author{
for
}

\section{Calabi-Yau threefolds and \\ $G_{2}$-manifolds}

\author{
Naichung Conan Leung
}

School of Mathematics, University of Minnesota, Minneapolis, MN 55454, USA.

\section{Introduction}

In the past two decades we have witnessed many fruitful interactions between mathematics and physics. One example is in the Donaldson-Floer theory for oriented four manifolds. Physical considerations lead to the discovery of the Seiberg-Witten theory which has profound impact to our understandings of four manifolds. Another example is in the mirror symmetry for Calabi-Yau manifolds. This duality transformation in the string theory leads to many surprising predictions in the enumerative geometry. 
String theory in physics studies a ten dimensional space-time $X \times \mathbb{R}^{3,1}$. Here $X$ a six dimensional Riemannian manifold with its holonomy group inside $S U$ (3), the so-called Calabi-Yau threefold. Certain parts of the mirror symmetry conjecture, as studied by Vafa's group, are specific for Calabi-Yau manifolds of complex dimension three. They include the Gopakumar-Vafa conjecture for the Gromov-Witten invariants of arbitrary genus, the OoguriVafa conjecture on the relationships between knot invariants and enumerations of holomorphic disks and so on. The key reason is they belong to a duality theory for $G_{2}$-manifolds. $G_{2}$-manifolds can be naturally interpreted as special Octonion manifolds [23]. For any Calabi-Yau threefold $X$, the seven dimensional manifold $X \times S^{1}$ is automatically a $G_{2}$-manifold because of the natural inclusion $S U(3) \subset G_{2}$.

In recent years, there are many studies of $G_{2}$-manifolds in M-theory including works of Archaya, Atiyah, Gukov, Vafa, Witten, Yau, Zaslow and many others (e.g. [1], [5], [13], [2]).

In the studies of the symplectic geometry of a Calabi-Yau threefold $X$, we consider unitary flat bundles over three dimensional (special) Lagrangian submanifolds $L$ in $X$. The corresponding geometry for a $G_{2}$-manifold $M$ is called the special $\mathbb{H}$-Lagrangian geometry (or C-geometry in [19]). where we consider Anti-Self-Dual (abbrev. ASD) bundles over four dimensional coassociative submanifolds, or equivalently special $\mathbb{H}$-Lagrangian submanifolds of type II [23], (abbrev. $\mathbb{H}-\mathrm{SLag}) C$ in $M$.

Counting ASD bundles over a fixed four manifold $C$ is the well-known theory of Donaldson differentiable invariants, Don $(C)$. Similarly, counting unitary flat bundles over a fixed three manifold $L$ is Floer's Chern-Simons homology theory, $H F_{C S}(L)$. When $C$ is a connected sum $C_{1} \#_{L} C_{2}$ along a homology three sphere, the relative Donaldson invariants Don $\left(C_{i}\right)$ 's take values in $H F_{C S}(L)$ and $D o n(C)$ can be recovered from individual pieces by a gluing theorem, $\operatorname{Don}(C)=\left\langle\operatorname{Don}\left(C_{1}\right) \text {, Don }\left(C_{2}\right)\right\rangle_{H F_{C S}(L)}$ (see e.g. [7]). Similarly when $L$ has a handlebody decomposition $L=L_{1} \#_{\Sigma} L_{2}$, each $L_{i}$ determines a Lagrangian subspace $\mathcal{L}_{i}$ in the moduli space $\mathcal{M}^{\text {flat }}(\Sigma)$ of unitary flat bundles over the Riemann surface $\Sigma$ and Atiyah conjectures that we can recover $H F_{C S}(L)$ from the Floer's Lagrangian intersection homology group of $\mathcal{L}_{1}$ and $\mathcal{L}_{2}$ in $\mathcal{M}^{\text {flat }}(\Sigma), H F_{C S}(L)=H F_{\text {Lag }}^{\mathcal{M}^{\text {flat }}(\Sigma)}\left(\mathcal{L}_{1}, \mathcal{L}_{2}\right)$. Such algebraic structures in the Donaldson-Floer theory can be formulated as a Topological Quantum Field Theory (abbrev. TQFT), as defined by Segal and Atiyah [3].

In this paper, we propose a construction of a TQFT by counting ASD bundles over four dimensional $\mathbb{H}$-SLag $C$ in any closed (almost) $G_{2}$-manifold 
$M$. We call these $\mathbb{H}-S L a g$ cycles and they can be identified as zeros of a naturally defined closed one form on the configuration space of topological cycles. We expect to obtain a homology theory $H_{C}(M)$ by applying the construction in the Witten's Morse theory. When $M$ is non-compact with an asymptotically cylindrical end, $X \times[0, \infty)$, then the collection of boundary data of relative $\mathbb{H}-S L a g$ cycles determines a Lagrangian submanifold $\mathcal{L}_{M}$ in the moduli space $\mathcal{M}^{S L a g}(X)$ of special Lagrangian cycles in the Calabi-Yau threefold $X$.

When we decompose $M=M_{1} \#_{X} M_{2}$ along an infinite asymptotically cylindrical neck, it is reasonable to expect to have a gluing formula,

$$
H_{C}(M)=H F_{\text {Lag }}^{\mathcal{M}^{S L a g}(X)}\left(\mathcal{L}_{M_{1}}, \mathcal{L}_{M_{2}}\right) .
$$

The main technical difficulty in defining this TQFT rigorously is the compactness issue for the moduli space of $\mathbb{H}-$ SLag cycles in $M$. We do not know how to resolve this problem and our homology groups are only defined in the formal sense (and physical sense?).

\section{$2 \quad G_{2}$-manifolds and $\mathbb{H}-\mathrm{SLag}$ geometry}

We first review some basic definitions and properties of $G_{2}$-geometry, see [19] for more details.

Definition 1. A seven dimensional Riemannian manifold $M$ is called a $G_{2^{-}}$ manifold if the holonomy group of its Levi-Civita connection is inside $G_{2} \subset$ $S O(7)$.

The simple Lie group $G_{2}$ can be identified as the subgroup of $S O(7)$ consisting of isomorphism $g: \mathbb{R}^{7} \rightarrow \mathbb{R}^{7}$ preserving the linear three form $\Omega$,

$$
\Omega=f^{1} f^{2} f^{3}-f^{1}\left(e^{1} e^{0}+e^{2} e^{3}\right)-f^{2}\left(e^{2} e^{0}+e^{3} e^{1}\right)-f^{3}\left(e^{3} e^{0}+e^{1} e^{2}\right),
$$

where $e^{0}, e^{1}, e^{2}, e^{3}, f^{1}, f^{2}, f^{3}$ is any given orthonormal frame of $\mathbb{R}^{7}$. Such a three form, or up to conjugation by elements in $G L(7, \mathbb{R})$, is called positive, and it determines a unique compatible inner product on $\mathbb{R}^{7}[6]$.

Gray [12] shows that $G_{2}$-holonomy of $M$ can be characterized by the existence of a positive harmonic three form $\Omega$.

Definition 2. A seven dimensional manifold $M$ equipped with a positive closed three form $\Omega$ is called an almost $G_{2}$-manifold. 
Remark: The relationship between $G_{2}$-manifolds and almost $G_{2}$-manifolds is analogous to the relationship between Kahler manifolds and symplectic manifolds. Namely we replace a parallel non-degenerate form by a closed one.

For example, suppose that $X$ is a complex three dimensional Kähler manifold with a trivial canonical line bundle, i.e. there exists a nonvanishing holomorphic three form $\Omega_{X}$. Yau's celebrated theorem says that there is a Kähler form $\omega_{X}$ on $X$ such that the corresponding Kahler metric has holonomy in $S U(3)$, i.e. a Calabi-Yau threefold. In particular both $\Omega_{X}$ and $\omega_{X}$ are parallel forms. Then the product $M=X \times S^{1}$ is a $G_{2}$-manifold with

$$
\Omega=\operatorname{Re} \Omega_{X}+\omega_{X} \wedge d \theta
$$

Conversely, one can prove, using Bochner arguments, every $G_{2}$-metric on $X \times S^{1}$ must be of this form. More generally, if $\omega_{X}$ is a general Kähler form on $X$, then $\left(X \times S^{1}, \Omega\right)$ is an almost $G_{2}$-manifold and the converse is also true.

Next we quickly review the geometry of $\mathbb{H}-$ SLag cycles in an almost $G_{2^{-}}$ manifold (see [19]).

Definition 3. An orientable four dimensional submanifold $C$ in an almost $G_{2}$-manifold $(M, \Omega)$ is called a coassociative submanifold, or simply a $\mathbb{H}$ SLag, if the restriction of $\Omega$ to $C$ is identically zero,

$$
\left.\Omega\right|_{C}=0
$$

If $M$ is a $G_{2}$-manifold, then any coassociative submanifold $C$ in $M$ is calibrated by $* \Omega$ in the sense of Harvey and Lawson [14], in particular, it is an absolute minimal submanifold in $M$. The normal bundle of any $\mathbb{H}-$ SLag $C$ can be naturally identified with the bundle of self-dual two forms on $C$. McLean [27] shows that infinitesimal deformations of any $\mathbb{H}-$ SLag are unobstructed and they are parametrized by the space of harmonic self-dual two forms on $C$, i.e. $H_{+}^{2}(C, \mathbb{R})$.

For example, if $S$ is a complex surface in a Calabi-Yau threefold $X$, then $S \times\{t\}$ is a $\mathbb{H}-\mathrm{SLag}$ in $M=X \times S^{1}$ for any $t \in S^{1}$. Notice that $H_{+}^{2}(S, \mathbb{R})$ is spanned by the Kahler form and the real and imaginary parts of holomorphic two forms on $S$, and the latter can be identified holomorphic normal vector fields along $S$ because of the adjunction formula and the Calabi-Yau condition on $X$. Thus all deformations of $S \times\{t\}$ in $M$ as $\mathbb{H}-$ SLag submanifolds are of the same form. Similarly, if $L$ is a three dimensional special 
Lagrangian submanifold in $X$ with phase $\pi / 2$, i.e. $\left.\omega\right|_{L}=\left.\operatorname{Re} \Omega_{X}\right|_{L}=0$, then $L \times S^{1}$ is also a $\mathbb{H}$-SLag in $M=X \times S^{1}$. Furthermore, all deformations of $L \times S^{1}$ in $M$ as $\mathbb{H}-$-SLag submanifolds are of the same form because $H_{+}^{2}\left(L \times S^{1}\right) \cong H^{1}(L)$, which parametrizes infinitesimal deformations of special Lagrangian submanifolds in $X$.

Definition 4. A H-SLag cycle in an almost $G_{2}$-manifold $(M, \Omega)$ is a pair $\left(C, D_{E}\right)$ with $C$ a $\mathbb{H}-S L a g$ in $M$ and $D_{E}$ an $A S D$ connection over $C$.

Remark: $\mathbb{H}-$ SLag cycles are supersymmetric cycles in physics as studied in [26]. Their moduli space admits a natural three form and a cubic tensor [19], which play the roles of the correlation function and the Yukawa coupling in physics.

We assume that the ASD connection $D_{E}$ over $C$ has rank one, i.e. a $U(1)$ connection. This avoids the occurrence of reducible connections, thus the moduli space $\mathcal{M}^{\mathbb{H}-S L a g}(M)$ of $\mathbb{H}$-SLag cycles in $M$ is a smooth manifold. It has a natural orientation and its expected dimension equals $b^{1}(C)$, the first Betti number of $C$. This is because the moduli space of $\mathbb{H}$-SLags has dimension equals $b_{+}^{2}(C)[27]$ and the existence of an ASD $U(1)$-connection over $C$ is equivalent to $H_{-}^{2}(C, \mathbb{R}) \cap H^{2}(C, \mathbb{Z}) \neq \phi$. The number $b^{1}(C)$ is responsible for twisting by a flat $U(1)$-connection.

For simplicity, we assume that $b^{1}(C)=0$, otherwise, one can cut down the dimension of $\mathcal{M}^{\mathbb{H}-S L a g}(M)$ to zero by requiring the ASD connections over $C$ to have trivial holonomy around loops $\gamma_{1}, \ldots, \gamma_{b^{1}}(C)$ in $C$ representing an integral basis of $H_{1}(C, \mathbb{Z})$. We plan to count the algebraic number of points in this moduli space $\# \mathcal{M}^{\mathbb{H}-S L a g}(M)$.

This number, in the case of $X \times S^{1}$, can be identified with a proposed invariant of Joyce [17] defined by counting rigid special Lagrangian submanifolds in any Calabi-Yau threefold. To explain this, we need the following proposition on the strong rigidity of product $\mathbb{H}-$ SLags.

Proposition 5. If $L \times S^{1}$ is a $\mathbb{H}-S L a g$ in $M=X \times S^{1}$ with $X$ a Calabi-Yau threefold, then any $\mathbb{H}-S L a g$ representing the same homology class must also be a product.

Proof: For simplicity we assume that the volume of the $S^{1}$ factor is unity, $\operatorname{Vol}\left(S^{1}\right)=1$. If $L \times S^{1}$ is a $\mathbb{H}-$ SLag in $M$ then $L$ is special Lagrangian submanifold in $X$ with phase $\pi / 2$, i.e. $\left.\operatorname{Re} \Omega_{X}\right|_{L}=\left.\omega\right|_{L}=0$. Suppose $C$ is another $\mathbb{H}$-SLag in $M$ representing the same homology class, we have $\operatorname{Vol}(C)=\operatorname{Vol}(L)$. If we write $C_{\theta}=C \cap(X \times\{\theta\})$ for any $\theta \in S^{1}$, then 
$\operatorname{Vol}\left(C_{\theta}\right) \geq \operatorname{Vol}(L)$, as $L$ is a calibrated submanifold in $X$. Furthermore the equality sign holds only if $C_{\theta}$ is also calibrated. In general we have

$$
\operatorname{Vol}(C) \geq \int_{S^{1}} \operatorname{Vol}\left(C_{\theta}\right) d \theta
$$

with the equality sign holds if and only if $C$ is a product with $S^{1}$. Combining these, we have

$$
\operatorname{Vol}(L)=\operatorname{Vol}(C) \geq \int_{S^{1}} \operatorname{Vol}\left(C_{\theta}\right) d \theta \geq \int_{S^{1}} \operatorname{Vol}(L) d \theta=\operatorname{Vol}(L) .
$$

Thus both inequalities are indeed equal. Hence $C=L^{\prime} \times S^{1}$ for some special Lagrangian submanifold $L^{\prime}$ in $X$.

Suppose $M=X \times S^{1}$ is a product $G_{2}$-manifold and we consider product $\mathbb{H}$-SLag $C=L \times S^{1}$ in $M$. From the above proposition, every $\mathbb{H}-$ SLag representing $[C]$ must also be a product. Since $b_{+}^{2}(C)=b^{1}(L)$, the rigidity of the HI-SLag $C$ in $M$ is equivalent to the rigidity of the special Lagrangian submanifold $L$ in $X$. When this happens, i.e. $L$ is a rational homology three sphere, we have $b^{2}(C)=0$ and

No. of ASD U(1)-bdl $/ C=\# H^{2}(C, \mathbb{Z})=\# H^{2}(L, \mathbb{Z})=\# H_{1}(L, \mathbb{Z})$.

Here we have used the fact that the first cohomology group is always torsion free. Thus the number of such $\mathbb{H}$-SLag cycles in $X \times S^{1}$ equals the number of special Lagrangian rational homology three spheres in a Calabi-Yau threefold $X$, weighted by $\# H_{1}(L, \mathbb{Z})$. Joyce [17] shows that with this particular weight, the numbers of special Lagrangians in any Calabi-Yau threefold behave well under various surgeries on $X$, and expects them to be invariants. Thus in this case, we have

$\# \mathcal{M}^{\mathbb{H}-S L a g}\left(X \times S^{1}\right)=$ Joyce's proposed invariant for \#SLag. in $X$.

In the next section, we will propose a homology theory, whose Euler characteristic gives $\# \mathcal{M}^{\mathbb{H}-S L a g}(M)$.

\section{$3 \quad$ Witten's Morse theory for $\mathbb{H}-$ SLag cycles}

We are going to use the parametrized version of $\mathbb{H}-S L a g$ cycles in any almost $G_{2}$-manifold $M$. We fix an oriented smooth four dimensional manifold $C$ and 
a rank $r$ Hermitian vector bundle $E$ over $C$. We consider the configuration space

$$
\mathcal{C}=\operatorname{Map}(C, M) \times \mathcal{A}(E),
$$

where $\mathcal{A}(E)$ is the space of Hermitian connections on $E$.

Definition 6. An element $\left(f, D_{E}\right)$ in $\mathcal{C}$ is called a parametrized $\mathbb{H}-S L a g$ cycles in $M$ if

$$
f^{*} \Omega=F_{E}^{+}=0,
$$

where the self-duality is defined using the pullback metric from $M$.

Instead of $A u t(E)$, the symmetry group $\mathcal{G}$ in our situation consists of gauge transformations of $E$ which cover arbitrary diffeomorphisms on $M$,

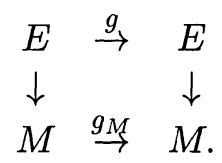

It fits into the following exact sequence,

$$
1 \rightarrow \operatorname{Aut}(E) \rightarrow \mathcal{G} \rightarrow \operatorname{Diff}(C) \rightarrow 1 .
$$

The natural action of $\mathcal{G}$ on $\mathcal{C}$ is given by

$$
g \cdot\left(f, D_{E}\right)=\left(f \circ g_{M}, g^{*} D_{E}\right),
$$

for any $\left(f, D_{E}\right) \in \mathcal{C}=\operatorname{Map}(C, M) \times \mathcal{A}(E)$. Notice that $\mathcal{G}$ preserves the set of parametrized $\mathbb{H}-\mathrm{SL}$ ag cycles in $M$.

The configuration space $\mathcal{C}$ has a natural one form $\Phi_{0}$ : At any $\left(f, D_{E}\right) \in \mathcal{C}$ we can identify the tangent space of $\mathcal{C}$ as

$$
T_{\left(f, D_{E}\right)} \mathcal{C}=\Gamma\left(C, f^{*} T_{M}\right) \times \Omega^{1}(C, a d(E)) .
$$

We define

$$
\Phi_{0}\left(f, D_{E}\right)(v, B)=\int_{C} \operatorname{Tr}\left[f^{*}\left(\iota_{v} \Omega\right) \wedge F_{E}+f^{*} \Omega \wedge B\right],
$$

for any $(v, B) \in T_{\left(f, D_{E}\right)} \mathcal{C}$.

Proposition 7. The one form $\Phi_{0}$ on $\mathcal{C}$ is closed and invariant under the action by $\mathcal{G}$. 
Proof: Recall that there is a universal connection $\mathbb{D}_{E}$ over $C \times \mathcal{A}(E)$ whose curvature $\mathbb{F}_{E}$ at a point $\left(x, D_{E}\right)$ equals,

$$
\begin{aligned}
\left.\mathbb{F}_{E}\right|_{\left(x, D_{E}\right)} & =\left(\mathbb{F}_{E}^{2,0}, \mathbb{F}_{E}^{1,1}, \mathbb{F}_{E}^{0,2}\right) \\
& \in \Omega^{2}(C) \otimes \Omega^{0}(\mathcal{A})+\Omega^{1}(C) \otimes \Omega^{1}(\mathcal{A})+\Omega^{0}(C) \otimes \Omega^{2}(\mathcal{A})
\end{aligned}
$$

with

$$
\mathbb{F}_{E}^{2,0}=F_{E}, \mathbb{F}_{E}^{1,1}(v, B)=B(v), \mathbb{F}_{E}^{0,2}=0,
$$

where $v \in T_{x} C$ and $B \in \Omega^{1}(C, a d(E))=T_{D_{E}} \mathcal{A}(E)$ (see e.g. [20]). The Bianchi identity implies that $\operatorname{Tr} \mathbb{F}_{E}$ is a closed form on $C \times \mathcal{A}(E)$. We also consider the evaluation map,

$$
\begin{gathered}
e v: C \times M a p(C, M) \rightarrow M \\
e v(x, f)=f(x) .
\end{gathered}
$$

It is not difficult to see that the pushforward of the differential form $e v^{*}(\Omega) \wedge$ $\operatorname{Tr} \mathbb{F}_{E}$ on $C \times M a p(C, M) \times \mathcal{A}(E)$ to $\operatorname{Map}(C, M) \times \mathcal{A}(E)$ equals $\Phi_{0}$, i.e.

$$
\Phi_{0}=\int_{C} e v^{*}(\Omega) \wedge \operatorname{Tr} \mathbb{F}_{E} .
$$

Therefore the closedness of $\Phi_{0}$ follows from the closedness of $\Omega$. It is also clear from this description of $\Phi_{0}$ that it is $\mathcal{G}$-invariant.

From this proposition, we know that $\Phi_{0}=d \Psi_{0}$ locally for some function $\Psi_{0}$ on $\mathcal{C}$. As in the Chern-Simons theory, this function $\Psi_{0}$ can be obtained explicitly by integrating the closed one form $\Phi_{0}$ along any path joining to a fixed element in $\mathcal{C}$. When $M=X \times S^{1}$ and $C=L \times S^{1}$, this is essentially the functional used by Thomas in [30].

From now on, we assume that $E$ is a rank one bundle.

Lemma 8. The zeros of $\Phi_{0}$ are the same as parametrized $\mathbb{H}-S L a g$ cycles in $M$.

Proof: Suppose $\left(f, D_{E}\right)$ is a zero of $\Phi_{0}$. By evaluating it on various $(0, B)$, we have $f^{*} \Omega=0$, i.e. $f: C \rightarrow M$ is a parametrized $\mathbb{H}$-SLag. This implies that the map

$$
\lrcorner \Omega: T_{f(x)} M \rightarrow \Lambda^{2} T_{x}^{*} C
$$

has image equals $\Lambda_{+}^{2} T_{x}^{*} C$, for any $x \in C$. By evaluating $\Phi_{0}$ on various $(v, 0)$, we have $F_{E}^{+}=0$, i.e. $\left(f, D_{E}\right)$ is a parametrized $\mathbb{H}-$ SLag cycle in $M$. The converse is obvious. 
From above results, $\Phi_{0}$ descends to a closed one form on $\mathcal{C} / \mathcal{G}$, called $\Phi$. Locally we can write $\Phi=d \mathcal{F}$ for some function $\mathcal{F}$ whose critical points are precisely (unparametrized) $\mathbb{H}$-SLag cycles in $M$. Using the gradient flow lines of $\mathcal{F}$, we could formally define a Witten's Morse homology group, as in the famous Floer's theory. Roughly speaking one defines a complex $\left(\mathbf{C}_{*}, \partial\right)$, where $\mathbf{C}_{*}$ is the free Abelian group generated by critical points of $\mathcal{F}$ and $\partial$ is defined by counting the number of gradient flow lines between two critical points of relative index one.

Remark: The equations for the gradient flow are given by

$$
\frac{\partial f}{\partial t}=*\left(f^{*} \xi \wedge F_{E}\right), \frac{\partial D_{E}}{\partial t}=*\left(f^{*} \Omega\right),
$$

where $\xi \in \Omega^{2}\left(M, T_{M}\right)$ is defined by $\langle\xi(u, v), w\rangle=\Omega(u, v, w)$.

The equation

$$
\partial^{2}=0
$$

requires a good compactification of the moduli space of $\mathbb{H}-\mathrm{SLag}$ cycles in $M$, which we are lacking at this moment (see [31] however). We denote this proposed homology group as $H_{C}(M)$, or $H_{C}(M, \alpha)$ when $f_{*}[C]=\alpha \in$ $H_{4}(M, \mathbb{Z})$.

This homology group should be invariant under deformations of the almost $G_{2}$-metric on $M$ and its Euler characteristic equals,

$$
\chi\left(H_{C}(M)\right)=\# \mathcal{M}^{\mathbb{H}-S L a g}(M) .
$$

Like Floer homology groups, they measure the middle dimensional topology of the configuration space $\mathcal{C}$ divided by $\mathcal{G}$.

\section{TQFT of $\mathbb{H}-$ SLag cycles}

In this section we study complete almost $G_{2}$-manifold $M_{i}$ with asymptotically cylindrical ends and the behavior of $H_{C}(M)$ when a closed almost $G_{2}$-manifold $M$ decomposes into connected sum of two pieces, each with an asymptotically cylindrical end,

$$
M=M_{1} \# M_{2} .
$$

Nontrivial examples of compact $G_{2}$-manifolds are constructed by Kovalev [18] using such connected sum approach. The boundary manifold $X$ is necessary a Calabi-Yau threefold. We plan to discuss analytic aspects of $M_{i}$ 's in a future paper [24]. 
Each $M_{i}$ 's will define a Lagrangian subspace $\mathcal{L}_{M_{i}}$ in the moduli space of special Lagrangian cycles in $X$. Furthermore we expect to have a gluing formula expressing the above homology group for $M$ in terms of the Floer Lagrangian intersection homology group for the two Lagrangian subspaces $\mathcal{L}_{M_{1}}$ and $\mathcal{L}_{M_{2}}$,

$$
H_{C}(M)=H F_{\text {Lag }}^{\mathcal{M}^{S \operatorname{Lag}(X)}}\left(\mathcal{L}_{M_{1}}, \mathcal{L}_{M_{2}}\right) .
$$

These properties can be reformulated to give us a topological quantum field theory. To begin we have the following definition.

Definition 9. An almost $G_{2}$-manifold $M$ is called cylindrical if $M=X \times \mathbb{R}^{1}$ and its positive three form respect such product structure, i.e.

$$
\Omega_{0}=\operatorname{Re} \Omega_{X}+\omega_{X} \wedge d t
$$

$A$ complete almost $G_{2}$-manifold $M$ with one end $X \times[0, \infty)$ is called asymptotically cylindrical if the restriction of its positive three form equals to the above one for large $t$, up to a possible error of order $O\left(e^{-t}\right)$. More precisely the positive three form $\Omega$ of $M$ restricted to its end equals,

$$
\Omega=\Omega_{0}+d \zeta
$$

for some two form $\zeta$ satisfying $|\zeta|+|\nabla \zeta|+\left|\nabla^{2} \zeta\right|+\left|\nabla^{3} \zeta\right| \leq C e^{-t}$.

Remark: If $M$ is an almost $G_{2}$-manifold with an asymptotically cylindrical end $X \times[0, \infty)$, then $\left(X, \omega_{X}, \Omega_{X}\right)$ is a complex threefold with a trivial canonical line bundle, but the Kähler form $\omega_{X}$ might not be Einstein. This is so, i.e. a Calabi-Yau threefold, provided that $M$ is a $G_{2}$-manifold. We will simply write $\partial M=X$.

We consider $\mathbb{H}-$ SLags $C$ in $M$ which satisfy a Neumann condition at infinity. That is, away from some compact set in $M$, the immersion $f: C \rightarrow$ $M$ can be written as

$$
f: L \times[0, \infty) \rightarrow X \times[0, \infty)
$$

with $\partial f / \partial t$ vanishes at infinite [24]. A relative HH-SLag itself has asymptotically cylindrical end $L \times[0, \infty)$ with $L$ a special Lagrangian submanifold in $X$. A relative $\mathbb{H}-S L a g$ cycle in $M$ is a pair $\left(C, D_{E}\right)$ with $C$ a relative $\mathbb{H}$-SLag in $M$ and $D_{E}$ a unitary connection over $C$ with finite energy,

$$
\int_{C}\left|F_{E}\right|^{2} d v<\infty
$$


Any finite energy connection $D_{E}$ on $C$ induces a unitary flat connection $D_{E^{\prime}}$ on $L[7]$.

Such a pair $\left(L, D_{E^{\prime}}\right)$ of a unitary flat connection $D_{E^{\prime}}$ over a special Lagrangian submanifold $L$ in a Calabi-Yau threefold $X$ is called a special $L a$ grangian cycle in $X$. Their moduli space $\mathcal{M}^{\text {SLag }}(X)$ plays an important role in the Strominger-Yau-Zaslow Mirror Conjecture [29] or [22]. The tangent space to $\mathcal{M}^{S L a g}(X)$ is naturally identified with $H^{2}(L, \mathbb{R}) \times H^{1}\left(L, a d\left(E^{\prime}\right)\right)$. For line bundles over $L$, the cup product

$$
\cup: H^{2}(L, \mathbb{R}) \times H^{1}(L, \mathbb{R}) \rightarrow \mathbb{R}
$$

induces a symplectic structure on $\mathcal{M}^{\text {SLag }}(X)$ [15]. Using analytic results from [24] about asymptotically cylindrical manifolds, we can prove the following theorem.

Claim 10. Suppose $M$ is an asymptotically cylindrical (almost) $G_{2}$-manifold with $\partial M=X$. Let $\mathcal{M}^{\mathbb{H}-S L a g}(M)$ be the moduli space of rank one relative $\mathbb{H}-S L a g$ cycles in $M$. Then the map defined by the boundary values,

$$
b: \mathcal{M}^{\mathbb{H}-S L a g}(M) \rightarrow \mathcal{M}^{\text {SLag }}(X),
$$

is a Lagrangian immersion.

Sketch of the proof ([24]): For any closed Calabi-Yau threefold $X$ (resp. $G_{2}$-manifold $M$ ), the moduli space of rank one special Lagrangian submanifolds $L$ (resp. $\mathbb{H}-$-SLags $C$ ) is smooth [27] and has dimension $b^{2}(L)$ (resp. $b_{+}^{2}(C)$ ). The same holds true for complete manifold $M$ with a asymptotically cylindrical end $X \times[0, \infty)$, where $b_{+}^{2}(C)_{L^{2}}$ denote the dimension of $L^{2}$-harmonic self-dual two forms on a relative $\mathbb{H}-\mathrm{SLag} C$ in $M$.

The linearization of the boundary value map $\mathcal{M}^{\mathbb{H}-S L a g}(M) \rightarrow \mathcal{M}^{\text {SLag }}(X)$ is given by $H_{+}^{2}(C)_{L^{2}} \stackrel{\alpha}{\rightarrow} H^{2}(L)$. Similar for the connection part, where the boundary value map is given by $H^{1}(C)_{L^{2}} \stackrel{\beta}{\rightarrow} H^{1}(L)$. We consider the following diagram where each row is a long exact sequence of $L^{2}$-cohomology groups for the pair $(C, L)$ and each column in a perfect pairing.

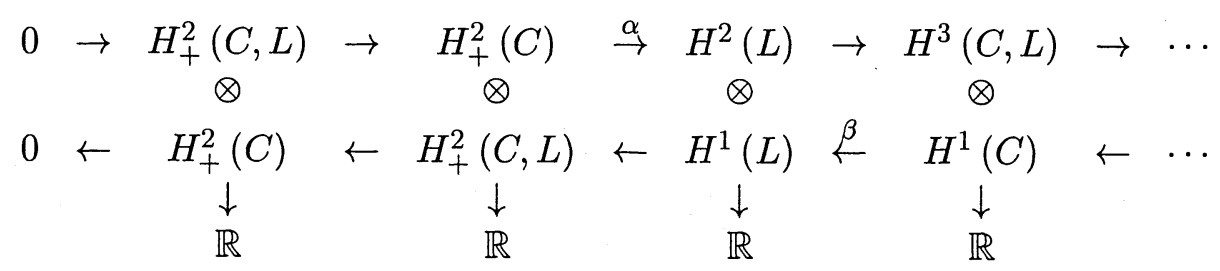


Notice that $H_{+}^{2}(C, L), H_{+}^{2}(C)$ and $H^{2}(L)$ parametrize infinitesimal deformation of $C$ with fixed $\partial C$, deformation of $C$ alone and deformation of $L$ respectively.

By simple homological algebra, it is not difficult to see that $\operatorname{Im} \alpha \oplus \operatorname{Im} \beta$ is a Lagrangian subspace of $H^{2}(L) \oplus H^{1}(L)$ with the canonical symplectic structure. Hence the result.

Remark: The deformation theory of conical special Lagrangian submanifolds is developed by Pacini in [28].

We denote the immersed Lagrangian submanifold $b\left(\mathcal{M}^{\mathbb{H}-S L a g}(M)\right)$ in $\mathcal{M}^{S L a g}(X)$ by $\mathcal{L}_{M}$. When $M$ decompose as a connected sum $M_{1} \#_{X} M_{2}$ along a long neck, as in Atiyah's conjecture on Floer Chern-Simons homology group [3], we expect to have an isomorphism,

$$
H_{C}(M) \cong H F_{\text {Lag }}^{\mathcal{M}^{\text {SLag }}(X)}\left(\mathcal{L}_{M_{1}}, \mathcal{L}_{M_{2}}\right) .
$$

More precisely, suppose $\Omega_{t}$ with $t \in[0, \infty)$, is a family of $G_{2}$-structure on $M_{t}=M$ such that as $t$ goes to infinite, $M$ decomposes into two components $M_{1}$ and $M_{2}$, each has an aymptotically cylindrical end $X \times[0, \infty)$. Then we expect that $\lim _{t \rightarrow \infty} H_{C}\left(M_{t}\right) \cong H F_{\text {Lag }}^{\mathcal{M}^{\text {SLag }}(X)}\left(\mathcal{L}_{M_{1}}, \mathcal{L}_{M_{2}}\right)$. We summarize these structures in the following table:

\begin{tabular}{|c||c|c|}
\hline Manifold: & (almost) $G_{2}$-manifold, $M^{7}$ & (almost) CY threefold, $X^{6}$ \\
\hline SUSY Cycles: & $\mathbb{H}$-SLag. submfds.+ ASD bdl & SLag submfds.+ flat bdl \\
\hline Invariant: & Homology group, $H_{C}(M)$ & Fukaya category, Fuk $\left(\mathcal{M}^{\text {SLag }}(X)\right)$. \\
\hline
\end{tabular}

These associations can be formalized to form a TQFT [4]. Namely we associate an additive category $F(X)=F u k\left(\mathcal{M}^{\text {SLag }}(X)\right)$ to a closed almost Calabi-Yau threefold $X$, a functor $F(M): F\left(X_{0}\right) \rightarrow F\left(X_{1}\right)$ to an almost $G_{2}$-manifold $M$ with asymptotically cylindrical ends $X_{1}-X_{0}=X_{1} \cup \bar{X}_{0}$. They satisfy

(i) $F(\phi)=$ the additive tensor category of vector spaces $((V e c))$,

(ii) $\quad F\left(X_{1} \amalg X_{2}\right)=F\left(X_{1}\right) \otimes F\left(X_{2}\right)$.

For example, when $M$ is a closed $G_{2}$-manifold, that is a cobordism between empty manifolds, then we have $F(M):((V e c)) \rightarrow((V e c))$ and the image of the trivial bundle is our homology group $H_{C}(M)$. 


\section{More TQFTs}

Notice that all TQFTs we propose in this paper are formal mathematical constructions. Besides the lack of compactness for the moduli spaces, the obstruction issue is also a big problem if we try to make these theories rigorous. This problem is explained to the author by a referee.

There are other TQFTs naturally associated to Calabi-Yau threefolds and $G_{2}$-manifolds but (1) they do not involve nontrivial coupling between submanifolds and bundles and (2) new difficulties arise because of corresponding moduli spaces for Calabi-Yau threefolds have virtual dimension zero and could be singular. They are essentially in the paper by Donaldson and Thomas [9].

\section{TQFT of associative cycles}

We assume that $M$ is a $G_{2}$-manifold, i.e. $\Omega$ is parallel rather than closed. Three dimensional submanifolds $A$ in $M$ calibrated by $\Omega$ is called associative submanifolds and they can be characterized by $\left.\chi\right|_{A}=0$ ([14]) where $\chi \in \Omega^{3}\left(M, T_{M}\right)$ is defined by $\langle w, \chi(x, y, z)\rangle=* \Omega(w, x, y, z)$. We define a parametrized $A$-cycle to be a pair $\left(f, D_{E}\right) \in \mathcal{C}_{A}=\operatorname{Map}(A, M) \times \mathcal{A}(E)$, with $f: A \rightarrow M$ a parametrized A-submanifold and $D_{E}$ is a unitary flat connection on a Hermitian vector bundle $E$ over $A$. There is also a natural $\mathcal{G}$-invariant closed one form $\Phi_{A}$ on $\mathcal{C}_{A}$ given by

$$
\Phi_{A}\left(f, D_{E}\right)(v, B)=\int_{A} \operatorname{Tr} F_{E} \wedge B+\left\langle f^{*} \chi, v\right\rangle_{T_{M}},
$$

for any $(v, B) \in \Gamma\left(A, f^{*} T_{M}\right) \times \Omega^{1}(A, a d(E))=T_{\left(f, D_{E}\right)} \mathcal{C}_{A}$. Its zero set is the moduli space of A-cycles in $M$. As before, we could formally apply arguments in Witten's Morse theory to $\Phi_{A}$ and define a homology group $H_{A}(M)$.

The corresponding category associated to a Calabi-Yau threefold $X$ would be the Fukaya-Floer category of the moduli space of unitary flat bundles over holomorphic curves in $X$, denote $\mathcal{M}^{\text {curve }}(X)$. We summarize these in the following table:

\begin{tabular}{|c||c|c|}
\hline Manifold: & $G_{2}$-manifold, $M^{7}$ & CY threefold, $X^{6}$ \\
\hline SUSY Cycles: & A-submfds. +flat bundles & Holomorphic curves+ flat bundles \\
\hline Invariant: & Homology group, $H_{A}(M)$ & Fukaya category, Fuk $\left(\mathcal{M}^{\text {curve }}(X)\right)$. \\
\hline
\end{tabular}




\section{TQFT of Donaldson-Thomas bundles}

We assume that $M$ is a seven manifold with a $G_{2}$-structure such that its positive three form $\Omega$ is co-closed, rather than closed, i.e. $d \Theta=0$ with $\Theta=* \Omega$. In [9] Donaldson and Thomas introduce a first order Yang-Mills equation for $G_{2}$-manifolds,

$$
F_{E} \wedge \Theta=0
$$

Their solutions are the zeros of the following gauge invariant one form $\Phi_{D T}$ on $\mathcal{A}(E)$,

$$
\Phi_{D T}\left(D_{E}\right)(B)=\int_{M} \operatorname{Tr}\left[F_{E} \wedge B\right] \wedge \Theta
$$

for any $B \in \Omega^{1}(M, a d(E))=T_{D_{E}} \mathcal{A}(E)$. This one form $\Phi_{D T}$ is closed because of $d \Theta=0$. As before, we can formally define a homology group $H_{D T}(M)$. The corresponding category associated to a Calabi-Yau threefold $X$ should be the Fukaya-Floer category of the moduli space of Hermitian Yang-Mills connections over $X$, denote $\mathcal{M}^{H Y M}(X)$. Again we summarize these in a table:

\begin{tabular}{|c||c|c|}
\hline Manifold: & $G_{2}$-manifold, $M^{7}$ & CY threefold, $X^{6}$ \\
\hline SUSY Cycles: & DT-bundles & Hermitian YM-bundles \\
\hline Invariant: & Homology group, $H_{D T}(M)$ & Fukaya category, Fuk $\left(\mathcal{M}^{H Y M}(X)\right)$. \\
\hline
\end{tabular}

It is an interesting problem to understand the transformations of these TQFTs under dualities in M-theory.

Acknowledgments: This paper is partially supported by NSF/DMS-0103355. The author expresses his gratitude to J.H. Lee, R. Thomas, A. Voronov and $X$.W. Wang for useful discussions. The author also thank the referee for many useful comments. 


\section{References}

[1] B. S. Acharya, B. Spence, Supersymmetry and $M$ theory on 7-manifolds, [hep-th/0007213].

[2] M. Aganagic, C. Vafa, Mirror Symmetry and a $G_{2}$ Flop, [hepth/0105225].

[3] M. Atiyah, New invariants of three and four dimensional manifolds, in The Mathematical Heritage of Herman Weyl, Proc. Symp. Pure Math., 48, A.M.S. (1988), 285-299.

[4] M. Atiyah, Topological quantum field theories. Inst. Hautes Études Sci. Publ. Math. No. 68 (1988), 175-186 (1989).

[5] M. Atiyah, E. Witten, $M$-theory dynamics on a manifold of $G_{2}$ holonomy, [hep-th/0107177].

[6] R. Bryant, Metrics with exceptional holonomy, Ann. of Math. 126 (1987) 525-576.

[7] S. Donaldson, Floer homology group in Yang-Mills theory, Cambridge Univ. Press (2002).

[8] S. Donaldson, P. Kronheimer, The geometry of four-manifols, Oxford University Press, (1990).

[9] S. Donaldson, R. Thomas, Gauge theory in higher dimension, The Geometric Universe: Science, Geometry and the work of Roger Penrose, S.A. Huggett et al edited, Oxford Univ. Press (1988).

[10] K. Fukaya, Y.G. Oh, H. Ohta, K. Ono, Lagrangian intersection Floer theory - anomoly and obstruction, to appear in International Press.

[11] R. Gopakumar, C. Vafa, M-theory and topological strings - II, [hepth/9812127].

[12] A. Gray, Vector cross products on manifolds, Trans. Amer. Math. Soc. 141 (1969) 465-504.

[13] S. Gukov, S.-T. Yau, E. Zaslow, Duality and Fibrations on $G_{2}$ Manifolds, [hep-th/0203217].

[14] R. Harvey, B. Lawson, Calibrated geometries, Acta Math. 148 (1982), $47-157$. 
[15] N. Hitchin, The moduli space of special Lagrangian submanifolds. Dedicated to Ennio DeGiorgi. Ann. Scuola Norm. Sup. Pisa Cl. Sci. (4) 25 (1997), no. 3-4, 503-515 (1998). [dg-ga/9711002].

[16] N. Hitchin, The geometry of three forms in 6 and 7 dimensions, J. Differential Geom. 55 (2000), no. 3, 547-576. [math.DG/0010054].

[17] D. Joyce, On counting special Lagrangian homology 3-spheres, [hepth/9907013].

[18] A. Kovalev, Twisted connected sums and special Riemannian holonomy, [math.DG/0012189].

[19] J.H. Lee, N.C. Leung, Geometric structures on $G_{2}$ and Spin(7)manifolds, [math.DG/0202045].

[20] N.C. Leung, Symplectic structures on gauge theory, Comm. Math. Phys., 193 (1998) 47-67.

[21] N.C. Leung, Mirror symmetry without corrections, [math.DG/0009235].

[22] N.C. Leung, Geometric aspects of mirror symmetry, to appear in the proceeding of ICCM 2001, [math.DG/0204168].

[23] N.C. Leung, Riemannian geometry over different normed division algebras, preprint 2002.

[24] N.C. Leung, in preparation.

[25] N.C. Leung, S.Y. Yau, E. Zaslow, From special Lagrangian to Hermitian-Yang-Mills via Fourier-Mukai transform, to appear in Adv. Thero. Math. Phys.. [math.DG/0005118].

[26] M. Marino, R. Minasian, G. Moore, and A. Strominger, Nonlinear Instantons from supersymmetric p-Branes, [hep-th/9911206].

[27] R. McLean, Deformations of calibrated submanifolds, Comm. Analy. Geom., 6 (1998) 705-747.

[28] T. Pacini, Deformations of Asymptotically Conical Special Lagrangian Submanifolds. Preprint 2002. [math.DG/0207144].

[29] A. Strominger, S.-T. Yau, and E. Zaslow, Mirror Symmetry is TDuality, Nuclear Physics B479 (1996) 243-259; [hep-th/9606040].

[30] R. P. Thomas, Moment maps, monodromy and mirror manifolds. In "Symplectic geometry and mirror symmetry", Proceedings of the 4th KIAS Annual International Conference, Seoul. Eds. K. Fukaya, Y.-G. Oh, K. Ono and G. Tian. World Scientific, 2001. 
[31] G. Tian, Gauge theory and calibrated geometry. I. Ann. of Math. (2) 151 (2000), no. 1, 193-268. 